\title{
Stretched quantum membranes
}

\author{
Jonas Björnsson ${ }^{1}$ and Stephen Hwang ${ }^{2}$ \\ Department of Physics \\ Karlstad University \\ SE-651 88 Karlstad, Sweden
}

\begin{abstract}
We study bosonic and space-time supersymmetric membranes with small tensions corresponding to stretched configurations. Using a generalized lightcone gauge, one may set up a perturbation theory around configurations having zero tension. We will show, by explicit construction to all orders in perturbation theory, that these membrane configurations are canonically equivalent, and thereby solvable, to string-like configurations with string excitations transverse to the stretched direction. At the quantum level, it is shown that there exists an ordering such that equivalence by unitary transformations is achieved. Consistency requires the critical dimensions 27 and 11 for the bosonic and supersymmetric cases, respectively. The mass spectrum is determined to any order. It is discrete and contains massless exitations. The ground state is purely string-like, whereas excited string-like states split through the perturbation into an infinite set of states with equal or lower energies.
\end{abstract}

\footnotetext{
1 jonas.bjornsson@kau.se

${ }^{2}$ stephen.hwang@kau.se
} 


\section{Introduction}

The relativistic membrane is, unlike string theory at the first quantized level, a highly complex theory. It is self-interacting and solutions to the equations of motion are, therefore, hard to find. Still, it is an interesting theory for many reasons. It is a theory in the same family as string theory, i.e. a geometrical theory where the action is proportional to the world-volume. As such it is interesting to study whether or not lessons learnt from string theory generalize, or if there are new and interesting features that are uncovered.

The membrane is also interesting from the point of M-theory [1, 2]. In 1] Mtheory was conjectured to be the supermembrane. A further connection between the two was established through the matrix model based on the work of [3, 4, 5] and the conjecture in [6]. In the former, a discretization of the area-preserving diffeomorphism algebra of the supermembrane action in lightcone gauge, gives a maximally supersymmetric $S U(N)$ matrix model. This matrix model should then in the large- $N$ limit recover the full dynamics of the supermembrane. The connection to the same matrix model proposed in [6], using the discrete lightcone approach, was further discussed in e.g. [7] and [8].

In view of the interest in the membrane and the complexity of the theory, any new results that may shed new light to its properties are highly valuable. In a previous paper [9] we formulated a perturbative approach to studying membranes around infinitely stretched configurations with zero tension. The perturbation parameter is the membrane tension. The unperturbed theory behaves as a string theory with string-like ${ }^{3}$ excitations transverse to the stretching. Consequently, one may exactly solve the unperturbed theory. String-like configurations have

\footnotetext{
${ }^{3}$ It is equivalent to the string theory apart from the fact that it depends on a third world-volume parameter. We use, therefore the terminology "string-like".
} 
also been found in the context of D-branes at strong coupling [10].

In [9] we proposed to solve the perturbation theory by canonical transformations, transforming the perturbed theory into the unperturbed one. In the present paper, we will prove that this proposal is indeed possible to implement to any order in perturbation theory. We will also treat the supermembrane, generalizing the result to this case as well. This implies that these membrane configurations, with small tensions, are classically equivalent to string-like theories and, therefore, completely solvable in terms of string-like solutions. Our results hold for open, semi-open and closed membranes.

Having shown the canonical equivalence at the classical level makes it possible to address the problem of quantization. We will show that it is possible to define a specific ordering such that the canonical transformations become unitary ones. Then the quantum theory requires, to any order in perturbation theory, the critical dimensions $D=27$ and $D=11$ for the bosonic membrane and supermembrane, respectively.

Evidence for these critical dimensions are indirectly present through the double-dimensional reduction [11, was further investigated for the supermembrane at the massless [12] and first massive level [13], and discussed in connection with the BRST symmetry in [14]. In [15, 16] these critical dimensions were found using Weyl-ordering and point-splitting regularization. In our case the critical dimensions arise from requirement that the $(D-1)$-dimensional Lorentz symmetry is non-anomalous. Indirectly, it is an implication that a two-dimensional subalgebra of the three-parameter reparametrization symmetry is non-anomalous for the critical dimensions to any order in perturbation theory. It is still an open question if our construction yields membranes that have full Lorentz invariance and reparametrization invariance at the quantum level. 
We can construct, using the unitary transformations and to any order in perturbation theory, an infinite set of physical states that diagonalizes the Hamiltonian. We will determine the spectrum to any order in perturbation theory. The spectrum is discrete and contains massless excitations. This is the case for both the bosonic and the supersymmetric membrane. The unperturbed spectrum is the usual string spectrum where each excited level is infinitely degenerate. The perturbation will break the degeneracy creating, in addition to the string-like states, an infinite set of states with lower mass. In particular, we will show that the mass splitting is the same for any order.

To arrive at this result, we have implicitly assumed a certain class of boundary conditions. The spectrum will depend crucially on this. In particular, for the supermembrane our choice seems to be the only one yielding a massless groundstate. Furthermore, it cannot be ruled out that for other boundary conditions it may be possible to have a continous spectrum.

Our results for the stretched membrane may be compared to results found in the matrix model approximation. For example, it has been shown that the bosonic membrane has a discrete spectrum [17, 18]. For the supermembrane one has, in general, a continous spectrum [18]. However, in [19] it was shown that for the $S U(2)$ supersymmetric matrix model there exists different possibilities. One possibility is a purely discrete spectrum and another is a spectrum which has a continous as well as a discrete part. The continous mass spectrum for the supermembrane can be understood in terms of the existence of infinitely thin tubes or stretches in the membrane surface [18]. For the bosonic case there is an infinite potential barrier preventing such effects. It has been proposed that the pinching of surfaces implies that supermembranes are second quantized from the outset. 
In our approach we will see that there is a barrier against pinching. The stretched membranes will always have a minimum width or circumference. This is a consequence of our particular gauge choice, keeping the string-like tension of the unperturbed theory fixed. Our results are consistent with the matrix model calculations for the bosonic case. For the supersymmetric case our results disagree with the matrix model calculations discussed above. This is true even for the case when the matrix model gives a discrete spectrum, since then there are no massless states [19. The disagreement could mean that our assumption regarding boundary conditions are not compatible with matrix model ones. It could also imply that the matrix model calculations probe the theory outside the weak tension regime, in which our perturbation theory is defined. Further investigations are certainly needed to answer these questions.

It is clear that in our perturbative approach we do not see any interactions between the string-like excitations. Interactions could arise as non-perturbative effects in our treatment. In a generic membrane configuration one may find stretched configurations in parts of the membrane surface eg. in thin tubes connecting different sections of the membrane, or in spikes that are attached to the surface. In these parts of the surface the excitations would, according to our results here, be purely string-like. An attractive interpretation of the string-like excitations would be as the elementary excitations of the membrane. In particular, the spikes could be thought of as in- and outgoing asymptotic string-like states. The non-stretched parts of the membrane surface correspond to sections of the surface in which interactions could take place.

The paper is organized as follows. In section two we make a short review of our formulation in 9] yielding a perturbation theory around infinitely stretched configurations. In section three we fix the gauge completely by using a generalized 
lightcone gauge and show the canonical equivalence to all orders in perturbation theory. We generalize our result to the supermembrane in section four. In section five we use the results in the previous sections to define a quantum theory which is unitarily equivalent to a string-like theory. The paper is concluded in the last section. In appendix A we give a few conventions and in appendix B we present some calculations on a toy model, that captures some of the relevant features of the membrane.

\section{The basic formalism and previous results}

The Dirac action [20] of the membrane is

$$
S=-T_{m} \int d^{3} \xi\left[-\operatorname{det}\left(\partial_{i} X_{U} \partial_{j} X^{U}\right)\right]^{1 / 2},
$$

where we use the mostly plus convension of the metric, $U=0, \ldots, D-1$ and $i, j=0,1,2 . \xi^{i}$ parametrize the world volume where $\xi^{0}$ is the time-like parameter. This action has three constraints, corresponding to reparametrization invariance of the world-volume.

By choosing a partial gauge $\chi \equiv X^{D-2}-\frac{1}{\sqrt{g}} \xi^{2} \approx 0$, where $g$ is a constant, the Hamiltonian may be put into the form 9

$$
\begin{aligned}
H & =\int d^{2} \xi \phi_{2} \\
& =H_{0}+g H_{1},
\end{aligned}
$$

with first-class constraints

$$
\begin{aligned}
\phi_{1} & =\mathcal{P} \partial_{1} X \approx 0 \\
\phi_{2} & =\frac{1}{2}\left\{\mathcal{P}^{2}+\left(\partial_{1} X\right)^{2}\right. \\
& \left.+g\left[\left(\partial_{1} X\right)^{2}\left(\partial_{2} X\right)^{2}+\left(\mathcal{P} \partial_{2} X\right)^{2}-\left(\partial_{1} X \partial_{2} X\right)^{2}\right]\right\} \approx 0 .
\end{aligned}
$$


All scalar products are now in the $D-1$ dimensional space-time $\mu=0, \ldots, D-$ $3, D-1$. We have, as can be seen from eqs. (2.2), (2.3) and (2.4) for $g \ll 1$, a perturbation theory, where the unperturbed $g=0$ limit yields a string-like theory with string-like tension ${ }^{4}, T_{s}=1$. In arriving at this formulation, we have taken $g=\left(\frac{T_{m}}{T_{s}}\right)^{2}$ so that $T_{m}=\sqrt{g} \ll 1=T_{s}$. Thus, $g \rightarrow 0$ is a zero-tension limit of the membrane such that the string tension in the $\xi^{1}$-direction remains non-zero. As the gauge choice $X^{D-2}-\frac{1}{\sqrt{g}} \xi^{2} \approx 0$ implies that $X^{D-2} \rightarrow \infty$ when $g \rightarrow 0$, we see that the membrane fills this space-direction completely. Thus, it is stretched in this direction. If the membrane is closed in the $\xi^{2}$-direction, the gauge-choice is consistent for $X^{D-2}$ being periodic. We will mostly consider a membrane that is periodic in both parameters i.e. have a torus topology. Other possibilites may be treated analogously and the results for these cases are indicated.

In 9] we studied the Hamiltonian defined in eq. (2.2). We were able to show the existence of canonical transformations that transformed the perturbed Hamiltonian to the unperturbed one to any order in perturbation theory. Since the gauge was only partially fixed, the effect of the transformations on the remaining constraints was not considered.

The canonical transformations in [9] also produced additional terms that involved the fields evaluated at the boundary. These terms are potentially problematic, as they contain factors that are non-local in the the space-direction corresponding to the boundary and, hence, may contribute to the equations of motion away from the boundary. This was not properly realized in 9]. It can be seen that, to first order in perturbation theory, the explicit expression in [9] does not give such problems, as the boundary terms actually vanish by the

\footnotetext{
${ }^{4}$ The string-like tension has dimension, $(\text { length })^{-3}$, which is different from the ordinary string tension.
} 
constraints. This was an implication that, by modifying the transformation, it should be possible to avoid generating boundary terms. The situation at arbitrary order was, however, an open question. The transformations constructed here will not generate boundary terms for the open case.

\section{The Bosonic membrane}

We will in this section show that one can map the perturbed theory to the unperturbed, string-like, theory by a canonical transformation. We do this by completely fixing a gauge for the bosonic membrane action.

Let us begin with the constraints for the membrane action

$$
\begin{aligned}
& \phi_{0}=\frac{1}{2} \mathcal{P}^{2}+\frac{T_{m}^{2}}{2}\left[\left(\partial_{1} X\right)^{2}\left(\partial_{2} X\right)^{2}-\left(\partial_{1} X \cdot \partial_{2} X\right)^{2}\right] \approx 0 \\
& \phi_{1}=\mathcal{P} \partial_{1} X \approx 0 \\
& \phi_{2}=\mathcal{P} \partial_{2} X \approx 0 .
\end{aligned}
$$

Fix a gauge partially by using the lightcone gauge

$$
\begin{aligned}
& \chi_{1} \equiv X^{+}-\xi^{0} \approx 0 \\
& \chi_{2} \equiv \mathcal{P}^{+}-1 \approx 0
\end{aligned}
$$

where we have used the conventions in appendix A. From this procedure one can define a Hamiltonian from $\mathcal{P}^{-}$

$$
\begin{aligned}
H & =-\int d^{2} \xi \mathcal{P}^{-} \\
& =\frac{1}{2} \int d^{2} \xi\left\{\mathcal{P}^{2}+T_{m}\left[\left(\partial_{1} X\right)^{2}\left(\partial_{2} X\right)^{2}-\left(\partial_{1} X \cdot \partial_{2} X\right)^{2}\right]\right\},
\end{aligned}
$$

where the scalar products are in the $D-2$ space-like dimensions. One constraint remains ungauged

$$
\phi=\partial_{1} \mathcal{P} \cdot \partial_{2} X-\partial_{2} \mathcal{P} \cdot \partial_{1} X \approx 0
$$


To gauge fix this, and to define our perturbation theory, we choose

$$
\chi_{3} \equiv X^{D-2}-\frac{1}{\sqrt{g}} \xi^{2} \approx 0 .
$$

$X^{D-2}$ has to be compact if one considers a closed membrane in this direction. Choosing $g=T_{m}^{2}$ this gauge defines a perturbation theory for $g \ll 1$ around a string-like theory in the lightcone gauge

$$
\begin{aligned}
H & =H_{0}+g H_{1} \\
H_{0} & =\frac{1}{2} \int d^{2} \xi\left[\mathcal{P}^{2}+\partial_{1} X^{2}\right] \\
H_{1} & =\frac{1}{2} \int d^{2} \xi\left[\left(\partial_{1} X\right)^{2}\left(\partial_{2} X\right)^{2}+B^{2}-\left(\partial_{1} X \partial_{2} X\right)^{2}\right]
\end{aligned}
$$

where $B \equiv-\frac{1}{\sqrt{g}} \mathcal{P}^{D-2}$ with

$$
\partial_{1} B=\partial_{1} \mathcal{P} \cdot \partial_{2} X-\partial_{2} \mathcal{P} \cdot \partial_{1} X
$$

and all scalar products are now in $D-3$ dimensions. These equations will be the starting point for our analysis of the perturbation theory.

Let us make a change of basis by

$$
\begin{aligned}
\alpha^{I} & =\frac{1}{\sqrt{2}}\left(\mathcal{P}^{I}+\partial_{1} X^{I}\right) \\
\tilde{\alpha}^{I} & =\frac{1}{\sqrt{2}}\left(\mathcal{P}^{I}-\partial_{1} X^{I}\right) .
\end{aligned}
$$

Using the fundamental Poisson bracket

$$
\left\{X^{I}(\xi), \mathcal{P}^{I}\left(\xi^{\prime}\right)\right\}=\delta^{I J} \delta^{2}\left(\xi-\xi^{\prime}\right)
$$

one has

$$
\begin{aligned}
& \left\{\alpha^{I}(\xi), \alpha^{J}\left(\xi^{\prime}\right)\right\}=\frac{1}{2} \delta^{I J}\left(\partial_{1}-\partial_{1}^{\prime}\right) \delta^{2}\left(\xi-\xi^{\prime}\right) \\
& \left\{\tilde{\alpha}^{I}(\xi), \tilde{\alpha}^{J}\left(\xi^{\prime}\right)\right\}=-\frac{1}{2} \delta^{I J}\left(\partial_{1}-\partial_{1}^{\prime}\right) \delta^{2}\left(\xi-\xi^{\prime}\right) .
\end{aligned}
$$


We make a Fourier expansion of the $\xi^{1}$-dependence of the fields $\alpha^{I}$ and $\tilde{\alpha}^{I}$

$$
\begin{aligned}
\alpha^{I} & =\frac{1}{\sqrt{2 \pi}} \sum_{m} \alpha_{m}^{I} \exp \left[-i m \xi^{1}\right] \\
\tilde{\alpha}^{I} & =\frac{1}{\sqrt{2 \pi}} \sum_{m} \tilde{\alpha}_{m}^{I} \exp \left[i m \xi^{1}\right]
\end{aligned}
$$

where $\alpha_{m}^{I}$ and $\tilde{\alpha}_{m}^{I}$ depend on $\xi^{0}$ and $\xi^{2}$. The non-zero Poisson brackets of the Fourier coefficients are

$$
\begin{aligned}
& \left\{\alpha_{m}^{I}\left(\xi^{2}\right), \alpha_{n}^{J}\left(\xi^{\prime 2}\right)\right\}=-i m \delta_{m,-n} \delta^{I J} \delta\left(\xi^{2}-\xi^{\prime 2}\right) \\
& \left\{\tilde{\alpha}_{m}^{I}\left(\xi^{2}\right), \tilde{\alpha}_{n}^{J}\left(\xi^{\prime 2}\right)\right\}=-i m \delta_{m,-n} \delta^{I J} \delta\left(\xi^{2}-\xi^{\prime 2}\right) .
\end{aligned}
$$

Using eq. (3.12) we have the Fourier expansions

$$
\begin{aligned}
\mathcal{P}^{I} & =\frac{1}{2 \sqrt{\pi}} \sum_{n}\left(\alpha_{n}^{I} \exp \left[-i n \xi^{1}\right]+\tilde{\alpha}_{n}^{I} \exp \left[i n \xi^{1}\right]\right) \\
\partial_{1} X^{I} & =\frac{1}{2 \sqrt{\pi}} \sum_{n}\left(\alpha_{n}^{I} \exp \left[-i n \xi^{1}\right]-\tilde{\alpha}_{n}^{I} \exp \left[i n \xi^{1}\right]\right)
\end{aligned}
$$

Integrating the last equation, requiring $X^{I}$ to be periodic, yields

$$
X^{I}=q^{I}+\frac{i}{2 \sqrt{\pi}} \sum_{n \neq 0} \frac{1}{n}\left(\alpha_{n}^{I} \exp \left[-i n \xi^{1}\right]-\tilde{\alpha}_{n}^{I} \exp \left[i n \xi^{1}\right]\right)
$$

$\alpha_{0}^{I}=\tilde{\alpha}_{0}^{I}$. From the fundamental Poisson brackets we have

$$
\left\{q^{I}\left(\xi^{2}\right), \alpha_{0}^{J}\left(\xi^{\prime 2}\right)\right\}=\frac{1}{2 \sqrt{\pi}} \delta^{I J} \delta\left(\xi^{2}-\xi^{\prime 2}\right)
$$

The unperturbed Hamiltonian is of the string-like form

$$
H_{0}=\frac{1}{2} \sum_{m, I} \int d \xi^{2}\left(\alpha_{-m}^{I} \alpha_{m}^{I}+\tilde{\alpha}_{-m}^{I} \tilde{\alpha}_{m}^{I}\right)
$$


We may also determine $B$,

$$
\begin{aligned}
B & =\frac{1}{2 \sqrt{\pi}} \sum_{m, I}\left(\partial_{2} q^{I} \alpha_{m}^{I} \exp \left[-i m \xi^{1}\right]+\partial_{2} q^{I} \tilde{\alpha}_{m}^{I} \exp \left[i m \xi^{1}\right]\right) \\
& +\frac{i}{4 \pi} \sum_{n \neq 0, m, I} \frac{1}{n}\left(\alpha_{m}^{I} \partial_{2} \alpha_{n}^{I} \exp \left[-i(m+n) \xi^{1}\right]+\tilde{\alpha}_{m}^{I} \partial_{2} \alpha_{n}^{I} \exp \left[i(m-n) \xi^{1}\right]\right. \\
& \left.+\alpha_{m}^{I} \partial_{2} \tilde{\alpha}_{n}^{I} \exp \left[-i(m-n) \xi^{1}\right]+\tilde{\alpha}_{m}^{I} \partial_{2} \tilde{\alpha}_{n}^{I} \exp \left[i(m+n) \xi^{1}\right]\right) \\
& -\frac{i}{2 \pi} \sum_{m+n \neq 0, I} \frac{1}{m+n}\left(\alpha_{m}^{I} \partial_{2} \alpha_{n}^{I} \exp \left[-i(n+m) \xi^{1}\right]\right. \\
& \left.+\tilde{\alpha}_{m}^{I} \partial_{2} \tilde{\alpha}_{n}^{I} \exp \left[i(n+m) \xi^{1}\right]\right),
\end{aligned}
$$

up to a function $f\left(\xi^{2}\right)$. This function will depend on the fourier modes of our basic fields through boundary conditions. We will take this function to be zero. Consistency will restrict the possible choices of boundary conditions. Other choices will not change our results apart from the spectrum. At the end of section five, we will briefly discuss the case of a non-zero function and possible implications on the mass spectrum.

If one now studies the perodicity condition on $X^{-}$one finds

$$
\sum_{m, I}\left[\alpha_{-m}^{I} \alpha_{m}^{I}-\tilde{\alpha}_{-m}^{I} \tilde{\alpha}_{m}^{I}\right]=0
$$

which is also a sufficient condition for $B$ to be periodic. Using $B$ one can explicitely find the expression for $H_{1}$.

Let us now consider the problem that we should solve. We would like to find a canonical transformation that transforms the perturbed theory at an arbitrary order to the unperturbed one. As $g \ll 1$ it is sufficient to consider infinitesimal transformations. To first order in perturbation theory we should, therefore, find a generator $G_{1}$ of infinitesimal canonical transformations that satisfies

$$
H \rightarrow H^{\prime}=H+g\left\{H, G_{1}\right\}=H_{0}+\mathcal{O}\left(g^{2}\right)
$$


which implies

$$
\left\{H_{0}, G_{1}\right\}=-H_{1}
$$

As can be seen from the explicit expression of $H_{1}$ in eq. (3.7) it is composed of quartic terms in the Fourier modes. $H_{0}$ is quadratic so that $G_{1}$ will also be quartic. To next order in perturbation theory one should find a generator $G_{2}$ such that

$$
H \rightarrow H^{\prime}=H+g\left\{H, G_{1}\right\}+\frac{g^{2}}{2}\left\{\left\{H, G_{1}\right\}, G_{1}\right\}+g^{2}\left\{H, G_{2}\right\}=H_{0}+\mathcal{O}\left(g^{3}\right)(3
$$

which implies

$$
\left\{H_{0}, G_{2}\right\}=-\left\{H_{1}, G_{1}\right\}-\frac{1}{2}\left\{\left\{H_{0}, G_{1}\right\}, G_{1}\right\} \equiv-H_{2} .
$$

$G_{2}$ will essentially be a sum of terms of products of six Fourier modes. Continuing this order by order, the $N^{\prime}$ th order generator will satisfy an equation of the form

$$
\left\{H_{0}, G_{N}\right\}=-H_{N}
$$

$G_{N}$ will essentially be a sum of terms of products of $2(N+1)$ Fourier modes. Even though $H_{N}$ becomes more and more complicated as $N$ grows larger, it is still possible to prove that a solution exists. The reason is that $G_{N}$ is determined through its Poisson bracket with $H_{0}$, which has a very simple form.

Beginning with the first order, $H_{1}$ may in a compact way be written as

$$
H_{1}=\int d \xi^{2} \sum_{r}\left\{\mathcal{H}_{(r)}^{0}+\partial_{2} q_{I} \mathcal{H}_{(r)}^{I}+\partial_{2} q_{I} \partial_{2} q_{J} \mathcal{H}_{(r)}^{I J}\right\}
$$

$\mathcal{H}_{(r)}^{0}, \mathcal{H}_{(r)}^{I}$ and $\mathcal{H}_{(r)}^{I J}$ are sums of terms with products of four, three and two factors of $\alpha_{m}^{I}, \tilde{\alpha}_{m}^{I}$ and derivatives with respect to $\xi^{2}$ of these fields satisfying

$$
\left\{H_{0}, \mathcal{H}_{(r)}^{(\ldots)}\right\}=i r \mathcal{H}_{(r)}^{(\ldots)}
$$


We first consider the problem of getting rid of a term $\mathcal{H}_{(r)}^{0}$ in $H_{1}$. For $r$ different from zero it is simple to find a solution and it is

$$
G_{1}^{\text {part. }}=-\frac{1}{i r} \int d \xi^{2} \mathcal{H}_{(r)}^{0} .
$$

Consider now the case when $r=0$. Introduce a quantity $K$ satisfying

$$
\left\{H_{0}, K\right\}=-1
$$

Such a quantity exists. Let us construct it by first calculating the Poisson bracket between $\int d \xi^{2} k_{I} q^{I}$, where $k^{I}$ is a constant vector, and $H_{0}$

$$
\left\{\int d \xi^{2} \alpha_{I, 0}\left(\xi^{2}\right) \alpha_{0}^{I}\left(\xi^{2}\right), \int d \xi^{\prime 2} k_{J}\left(\xi^{\prime}\right) q^{J}\left(\xi^{\prime}\right)\right\}=-\frac{1}{\sqrt{\pi}} \int d \xi^{2} k_{I} \alpha_{0}^{I} .
$$

Thus, $K$ is given by

$$
K \equiv \sqrt{\pi} \frac{\int d \xi^{2} k_{I} q^{I}}{\int d \xi^{2} k_{I} \alpha_{0}^{I}}
$$

which is well-defined if the centre of mass momentum $P_{0}^{I}=\int d \xi^{2} \alpha_{0}^{I}$ is non-zero. As $P_{0}^{I}$ is a constant of motion this requirement restricts the initial state of the membrane. One can now use this operator to solve the terms where $r=0$ by

$$
G_{1}^{\text {part. }}=K \int d \xi^{2} \mathcal{H}_{(0)}^{0}
$$

Let us now focus on more complicated terms containing $q^{I}$ 's in $H_{1}$. Consider the term in $H_{1}$ containing one $q^{I}$. Let us make an ansatz of the solution for $r \neq 0$ as

$$
\tilde{G}_{1}^{\text {part. }}=-\frac{1}{i r} \int d \xi^{2} \partial_{2} q_{I} \mathcal{H}_{(r)}^{I}
$$

The Poisson bracket of this term with $H_{0}$ gives a term that is the sought for term. In addition, it gives a term

$$
-\frac{1}{i r} \int d \xi^{2}\left(-\frac{\partial_{2} \alpha_{I, 0}}{\sqrt{\pi}}\right) \mathcal{H}_{(r)}^{I} .
$$


This term is of the same kind as the one considered above, which we have shown how to compensate by a canonical transformation. We can, therefore, write down the solution to the term with one $q^{I}$ and $r \neq 0$,

$$
G_{1}^{\text {part. }}=-\frac{1}{i r} \int d \xi^{2} \partial_{2} q_{I} \mathcal{H}_{(r)}^{I}+\frac{1}{(i r)^{2}} \int d \xi^{2}\left(-\frac{\partial_{2} \alpha_{I, 0}}{\sqrt{\pi}}\right) \mathcal{H}_{(r)}^{I} .
$$

When $r$ is equal to zero one can proceed in the same way. The solution for this part of $G_{1}$ is

$$
G_{1}^{\text {part. }}=K \int d \xi^{2} \partial_{2} q_{I} \mathcal{H}_{(0)}^{I}+\frac{K^{2}}{2} \int d \xi^{2}\left(-\frac{\partial_{2} \alpha_{I, 0}}{\sqrt{\pi}}\right) \mathcal{H}_{(0)}^{I} .
$$

The third term in eq. 3.26), containing two factors of $q$, is solved in a completely analogous fashion. Let us conclude by presenting the solution to first order

$$
\begin{aligned}
G_{1} & =\int d \xi^{2} \sum_{r}\left\{( - \frac { 1 } { i r } ( 1 - \delta _ { r , 0 } ) + K \delta _ { r , 0 } ) \left(\mathcal{H}_{(r)}^{0}+\partial_{2} q_{I} \mathcal{H}_{(r)}^{I}\right.\right. \\
& \left.+\partial_{2} q_{I} \partial_{2} q_{J} \mathcal{H}_{(r)}^{I J}\right)+\left(\frac{1}{(i r)^{2}}\left(1-\delta_{r, 0}\right)+\frac{K^{2}}{2} \delta_{r, 0}\right) \\
& \times\left(\left(-\frac{\partial_{2} \alpha_{I, 0}}{\sqrt{\pi}}\right) \mathcal{H}_{(r)}^{I}+2\left(-\frac{\partial_{2} \alpha_{I, 0}}{\sqrt{\pi}}\right) \partial_{2} q_{J} \mathcal{H}_{(r)}^{I J}\right) \\
& \left.+2\left(-\frac{1}{(i r)^{3}}\left(1-\delta_{r, 0}\right)+\frac{K^{3}}{6} \delta_{r, 0}\right)\left(-\frac{\partial_{2} \alpha_{I, 0}}{\sqrt{\pi}}\right)\left(-\frac{\partial_{2} \alpha_{J, 0}}{\sqrt{\pi}}\right) \mathcal{H}_{(r)}^{I J}\right\} .
\end{aligned}
$$

We have by this expression constructed the solution of the generator of canonical transformation to first order. In the remaining part of this section we will show how one can generalize the solution (3.37) to get an expression to any order in perturbation theory. It is convenient to make a Fourier decomposition with 
respect to the $\xi^{2}$-dependence,

$$
\begin{aligned}
\alpha_{m}^{I} & =\frac{1}{\sqrt{2 \pi}} \sum_{n} \alpha_{m, n}^{I} \exp \left[i n \xi^{2}\right] \\
\tilde{\alpha}_{m}^{I} & =\frac{1}{\sqrt{2 \pi}} \sum_{n} \tilde{\alpha}_{m, n}^{I} \exp \left[i n \xi^{2}\right] \\
q^{I} & =\frac{1}{2^{3 / 2} \pi} \sum_{n} q_{n}^{I} \exp \left[i n \xi^{2}\right] .
\end{aligned}
$$

We have the following non-zero Poisson brackets

$$
\begin{aligned}
\left\{\alpha_{m, n}^{I}, \alpha_{p, q}^{J}\right\} & =-i m \delta_{m,-p} \delta_{n,-q} \delta^{I J} \\
\left\{\tilde{\alpha}_{m, n}^{I}, \tilde{\alpha}_{p, q}^{J}\right\} & =-i m \delta_{m,-p} \delta_{n,-q} \delta^{I J} \\
\left\{q_{m}^{I}, \alpha_{0, n}^{J}\right\} & =\delta_{m,-n} \delta^{I J} .
\end{aligned}
$$

From this one gets that the quantity $K$ has the form

$$
K=\frac{1}{2} \frac{k_{I} q_{0}^{I}}{k_{I} \alpha_{0,0}^{I}}
$$

To simplify the expressions, let us define a collective index $(a)=(I, n)$

$$
\alpha_{m, n}^{I}=\alpha_{m}^{(a)}
$$

In this notation we have the unperturbed Hamiltonian

$$
H_{0}=\frac{1}{2} \sum_{m,(a)}\left(\alpha_{m}^{(-a)} \alpha_{m}^{(a)}+\tilde{\alpha}_{m}^{(-a)} \tilde{\alpha}_{m}^{(a)}\right),
$$

where $(-a)=(I,-n)$ for $(a)=(I, n)$, and the first order Hamiltonian (cf. equation (3.26)

$$
H_{1}=\sum_{r}\left\{H_{(r)}^{0}+\sum_{(a)} q^{(-a)} H_{(r)}^{(a)}+\sum_{(a),(b)} q^{(-a)} q^{(-b)} H_{(r)}^{(a)(b)}\right\} .
$$

Consider the following general form of the Hamiltonian to $N$ 'th order

$$
H_{N}=\sum_{r} \sum_{i=0} \sum_{\left(a_{1}\right) \ldots\left(a_{i}\right)}\left\{q^{\left(-a_{1}\right)} \cdot \ldots \cdot q^{\left(-a_{i}\right)} H_{(r)}^{\left(a_{1}\right) \ldots\left(a_{i}\right)}\right\} .
$$


$H_{(r)}^{\left(a_{1}\right) \ldots\left(a_{i}\right)} \in \mathcal{F}$, which is the space of polynomials of $\alpha_{m_{p}}^{\left(a_{p}\right)}, \tilde{\alpha}_{m_{p}}^{\left(a_{p}\right)}$ and $\left(k_{I} \alpha_{0,0}^{I}\right)^{-1}$. Define also $\mathcal{G}$ as the space of polynomials of $\alpha_{m_{p}}^{\left(a_{p}\right)}, \tilde{\alpha}_{m_{p}}^{\left(a_{p}\right)},\left(k_{I} \alpha_{0,0}^{I}\right)^{-1}$ and $q^{I}$, so that $H_{N} \in \mathcal{G}$. We will prove below that this is the most general form of $H_{N}$ that can appear. It is true to first order, which can be seen from eq. (3.43). Before we proceed, let us note the following

$$
\begin{aligned}
\left\{q^{(a)}, f\right\} & \in \mathcal{F} \text { for } f \in \mathcal{F} \\
\left\{f, f^{\prime}\right\} & \in \mathcal{F} \text { for } f, f^{\prime} \in \mathcal{F} .
\end{aligned}
$$

This implies

$$
\left\{H_{N_{1}}^{\{1\}}, H_{N_{2}}^{\{2\}}\right\} \in \mathcal{G},
$$

where $H_{N_{i}}^{\{i\}} \in \mathcal{G}$. We also need to construct the canonical transformation which yields the Hamiltonian eq. (3.44). Proceeding in the same way as we did to first order, it is straightforward to construct the solution. One finds

$$
G_{N}=\sum_{r} G_{N}^{r}
$$

where

$$
\begin{aligned}
G_{N}^{r \neq 0} & =\sum_{i=0} \sum_{j=0}^{i} \sum_{\left(a_{1}\right) \ldots\left(a_{i}\right)}\left(\begin{array}{c}
p \\
j
\end{array}\right) \frac{(-1)^{j+1}}{(i r)^{j+1}}\left\{\left(-2 \alpha_{0}^{\left(-a_{1}\right)}\right) \cdot \ldots\right. \\
& \left.\times\left(-2 \alpha_{0}^{\left(-a_{j}\right)}\right) q^{\left(-a_{j+1}\right)} \cdot \ldots \cdot q^{\left(-a_{i}\right)} H_{(r)}^{\left(a_{1}\right) \ldots\left(a_{i}\right)}\right\} \\
G_{N}^{r=0} & =\sum_{i=0} \sum_{j=0}^{i} \sum_{\left(a_{1}\right) \ldots\left(a_{i}\right)}\left(\begin{array}{c}
p \\
j
\end{array}\right) \frac{K^{j}}{j !}\left\{\left(-2 \alpha_{0}^{\left(-a_{1}\right)}\right) \cdot \ldots \cdot\left(-2 \alpha_{0}^{\left(-a_{j}\right)}\right)\right. \\
& \left.\times q^{\left(-a_{j+1}\right)} \cdot \ldots \cdot q^{\left(-a_{i}\right)} H_{(0)}^{\left(a_{1}\right) \ldots\left(a_{i}\right)}\right\} .
\end{aligned}
$$

By inspection one sees that $G_{N} \in \mathcal{G}$. Therefore, by eq. (3.46)

$$
\left\{G_{N_{1}}^{\{1\}}, H_{N_{2}}^{\{2\}}\right\} \in \mathcal{G}
$$


where $G_{N_{1}}^{\{1\}} \in \mathcal{G}$ and $H_{N_{2}}^{\{2\}} \in \mathcal{G}$.

Let us now prove that eq. (3.44) is the most general form of the Hamiltonian. The proof is by induction. It is obviously true for the zeroth and first orders. $H_{N+1}$ is constructed by taking repeated Poisson brackets between generators $G_{i}$, where $i=1, \ldots, N$, and $H_{0}$. By the induction hypothesis $G_{i} \in \mathcal{G}$. Using equation (3.50) repeatedly proves the statement.

We have now shown that, to any order in perturbation theory, the Hamiltonian has the form given by eq. (3.44) and constructed the generators of canonical transformations, given by eqs. (3.47), (3.48) and (3.49), which transforms $H$ to $H_{0}$. We have here considered the closed case. Extending the analysis to the open case is straightforward. By an appropriate boundary condition, we will get a Fourier expansion w.r.t. $\xi^{1}$ with only one independent mode, $\alpha_{n}^{I}$. The proof we give above holds also for this case. In the next section we will extend our analysis to also hold for the completely gauge fixed supermembrane.

\section{The Supermembrane}

We will in this section generalize our results to the space-time supersymmetric membrane. Consider the following action proposed in [21]

$$
\begin{aligned}
S & =-T_{m} \int d^{3} \xi\left\{\sqrt{-h}+i \frac{1}{2} \epsilon^{i j k} \bar{\theta} \Gamma_{U V} \partial_{i} \theta\right. \\
& \left.\times\left[\Pi_{j}^{U} \partial_{k} X^{V}-\frac{1}{3} \bar{\theta} \Gamma^{U} \partial_{j} \theta \bar{\theta} \Gamma^{V} \partial_{k} \theta\right]\right\} .
\end{aligned}
$$

We specify the dimension to $D=11$, generalization to $D=4,5$ and 7 is straightforward at the classical level. Here $X^{U}$ are the 11 bosonic coordinates, $\theta^{\beta}$ are 32-component Majorana spinors, $\Pi_{i}^{U}=\partial_{i} X^{U}-i \bar{\theta} \Gamma^{U} \partial_{i} \theta, h$ is the determinant of the matrix $h_{i j}=\Pi_{i}^{U} \Pi_{j, U}, \Gamma^{U}$ are gamma matrices, $\Gamma_{U V}=\frac{1}{2}\left[\Gamma_{U}, \Gamma_{V}\right]$ and 
$\epsilon^{012}=-1$. This action is invariant under local fermionic transformations (kappa symmetry) and local reparametrizations. Let us first fix the kappa symmetry by

$$
\Gamma^{+} \theta=0,
$$

where we use the lightcone conventions presented in the appendix A. Using the explicit basis of $\Gamma^{+}$, given in Appendix A, one can see that $\theta^{2 \beta+1}=0$. We also rescale the other fermions by $\theta^{2 \beta}=2^{-1 / 4} \psi^{\beta}$. Passing on to the phasespace formulation, using the lightcone gauge, defined in eq. (3.3), yields the Hamiltonian [5]

$$
\begin{aligned}
H & =\int d^{2} \xi\left\{\frac{1}{2} \mathcal{P}^{2}+T_{m} i \epsilon^{a b} \psi \gamma_{A} \partial_{a} \psi \partial_{b} X^{A}\right. \\
& \left.+\frac{T_{m}^{2}}{2}\left[\left(\partial_{1} X\right)^{2}\left(\partial_{2} X\right)^{2}-\left(\partial_{1} X \cdot \partial_{2} X\right)^{2}\right]\right\} .
\end{aligned}
$$

We also have the remaining constraints

$$
\begin{aligned}
\phi & =\epsilon^{a b}\left(\partial_{a} \mathcal{P}^{A} \partial_{b} X_{A}+i \partial_{a} \psi \partial_{b} \psi\right) \approx 0 \\
G^{\beta} & =S^{\beta}-i \psi^{\beta} \approx 0,
\end{aligned}
$$

where $G^{\beta}$ are all second-class. Eliminating the second-class constraints gives rise to a non-zero Dirac bracket between the fermions

$$
\left\{\psi^{\beta}(\xi), \psi^{\gamma}\left(\xi^{\prime}\right)\right\}^{*}=\frac{1}{2 i} \delta^{\beta \gamma} \delta^{2}\left(\xi-\xi^{\prime}\right)
$$

We eliminate the bosonic constraint by imposing the gauge in eq. (3.6). Denote also $g=T_{m}^{2}$. Letting $g$ be small yields a perturbation theory where the 
Hamiltonian can be divided into three parts as

$$
\begin{aligned}
H & =H_{0}+\sqrt{g} H_{1}+g H_{2} \\
H_{0} & =\int d^{2} \xi\left\{\frac{1}{2}\left[\mathcal{P}^{2}+\left(\partial_{1} X\right)^{2}\right]+i \psi_{1} \partial_{1} \psi_{1}-i \psi_{2} \partial_{1} \psi_{2}\right\} \\
H_{1} & =\int d^{2} \xi i \epsilon^{a b} \psi \gamma_{I} \partial_{a} \psi \partial_{b} X^{I} \\
H_{2} & =\frac{1}{2} \int d^{2} \xi\left[\left(\partial_{1} X\right)^{2}\left(\partial_{2} X\right)^{2}+\tilde{B}^{2}-\frac{1}{2}\left(\partial_{1} X \partial_{2} X\right)^{2}\right],
\end{aligned}
$$

where $\tilde{B} \equiv-\frac{1}{\sqrt{g}} \mathcal{P}^{D-2}$ and

$$
\psi=\left(\begin{array}{l}
\psi^{1} \\
\psi^{2}
\end{array}\right)
$$

with

$$
\begin{aligned}
\partial_{1} \tilde{B} & =-\frac{1}{\sqrt{g}} \partial_{1} \mathcal{P}^{D-2} \\
& =\partial_{1} \mathcal{P} \cdot \partial_{2} X+i \partial_{1} \psi_{1} \partial_{2} \psi_{1}-\partial_{2} \mathcal{P} \cdot \partial_{1} X-i \partial_{2} \psi_{2} \partial_{1} \psi_{2}
\end{aligned}
$$

Note here the difference as compared to the bosonic membrane. Here we get order $\sqrt{g}$ corrections, which are terms with one $\xi^{2}$-derivative.

Let us proceed in the same manner as in the previous section. Change coordinates from $\left(\partial_{1} X^{I}, P^{I}\right)$ to $\left(\alpha^{I}, \tilde{\alpha}^{I}\right)$. Expanding these into Fourier modes yields in the end eqs. (3.14) and (3.16) with $\alpha_{0}^{I}=\tilde{\alpha}_{0}^{I}$. Similarly, for the fermions

$$
\begin{aligned}
\psi_{\beta}^{1} & =\frac{1}{2 \sqrt{\pi}} \sum_{n} \psi_{\beta, n}^{1} \exp \left[-i n \xi^{1}\right] \\
\psi_{\beta}^{2} & =\frac{1}{2 \sqrt{\pi}} \sum_{n} \psi_{\beta, n}^{2} \exp \left[i n \xi^{1}\right]
\end{aligned}
$$

where $\psi_{\beta, n}^{1}$ and $\psi_{\beta, n}^{2}$ depend on $\xi^{0}$ and $\xi^{2}$. The non-zero Poisson brackets are

$$
\begin{aligned}
& \left\{\psi_{\beta, m}^{1}, \psi_{\gamma, n}^{1}\right\}=-i \delta_{m,-n} \delta_{\beta \gamma} \delta\left(\xi^{2}-\xi^{2}\right) \\
& \left\{\psi_{\beta, m}^{2}, \psi_{\gamma, n}^{2}\right\}=-i \delta_{m,-n} \delta_{\beta \gamma} \delta\left(\xi^{2}-\xi^{\prime 2}\right)
\end{aligned}
$$


Inserting this into the unperturbed Hamiltonian yields

$$
H_{0}=\frac{1}{2} \sum_{m} \int d^{2} \xi\left[\left(\alpha_{-m}^{I} \alpha_{I, m}+\alpha_{-m}^{I} \alpha_{I, m}\right)+m\left(\psi_{-m}^{1} \psi_{m}^{1}+\psi_{-m}^{2} \psi_{m}^{2}\right)\right] .
$$

Before we proceed, let us determine the explicit expression of $\tilde{B}$, which we calculate by inserting the Fourier expansions of the different fields

$$
\begin{aligned}
\tilde{B} & =B+\frac{i}{4 \pi} \sum_{n, m}\left\{\psi_{m}^{1} \partial_{2} \psi_{n}^{1} \exp \left[-i(m+n) \xi^{1}\right]+\psi_{m}^{2} \partial_{2} \psi_{n}^{2} \exp \left[i(m+n) \xi^{1}\right]\right\} \\
& -\frac{i}{2 \pi} \sum_{n+m \neq 0} \frac{n}{n+m}\left\{\psi_{m}^{1} \partial_{2} \psi_{n}^{1} \exp \left[-i(m+n) \xi^{1}\right]\right. \\
& \left.+\psi_{m}^{2} \partial_{2} \psi_{n}^{2} \exp \left[i(m+n) \xi^{1}\right]\right\}
\end{aligned}
$$

where $B$ is given by eq. (3.19). The periodicity requires

$$
\sum_{m}\left[\alpha_{-m}^{I} \alpha_{I, m}+m \psi_{-m}^{1} \psi_{m}^{1}-\tilde{\alpha}_{-m}^{I} \tilde{\alpha}_{I, m}-m \psi_{-m}^{2} \psi_{m}^{2}\right]=0
$$

If we study the Poisson brackets between the unperturbed Hamiltonian and terms of the form

$$
\begin{aligned}
\mathcal{H}_{(r)}\left(\xi^{2}\right) & =C_{I_{1}, \ldots, I_{N}}^{\alpha_{N+1}, \ldots, \alpha_{R}}\left(n_{i}\right) \alpha_{n_{1}}^{I_{1}} \cdot \ldots \cdot \alpha_{n_{M}}^{I_{M}} \cdot \tilde{\alpha}_{n_{M+1}}^{I_{M+1}} \cdot \ldots \cdot \tilde{\alpha}_{n_{N}}^{I_{N}} \\
& \times \psi_{n_{N+1}, \alpha_{N+1}}^{1} \ldots \cdot \psi_{n_{P}, \alpha_{P}}^{1} \psi_{n_{P+1}, \alpha_{P+1}}^{2} \cdot \ldots \cdot \psi_{n_{R}, \alpha_{R}}^{2} \\
r & =\sum_{i=1}^{R} n_{i}
\end{aligned}
$$

one finds

$$
\left\{H_{0}, \mathcal{H}_{(r)}\right\}=\operatorname{ir}_{(r)}
$$

This also holds true for terms that involve derivatives with respect to $\xi^{2}$. Therefore, $\mathcal{H}_{(r)}$ satisfies the same crucial Poisson bracket as the corresponding expression for the bosonic case, eq. (3.27). We can then make a decomposition of different terms that can appear in $H_{N}$ in the same way as in eq. (3.44) where, 
in addition, $\mathcal{F}$ and $\mathcal{G}$ contains fermion modes $\psi_{\beta, n}^{i}$. Furthermore, the quantity $K$ defined in eq. (3.31) still satisfies the property eq. (3.29). This means that the construction of $G_{N}$ proceeds in exactly the same way as for the bosonic case with a general solution given by eqs. (3.47), (3.48) and (3.49). This generalizes also to the open case.

\section{Quantization}

Our considerations this far have been purely classical. In this section we will show that there exist, as far as we have been able to check, consistent quantum theories to any finite order in perturbation theory for stretched membranes in $D=27$ and $D=11$ for the bosonic and supersymmetric cases, respectively.

The preceeding sections show that the membrane is, in a particular gauge and within our perturbation theory, canonically equivalent to a string-like theory. This does not automatically mean that there is a quantum equivalence, due to ordering problems. Every canonical transformation will not correspond to a unitary transformation. Our approach is, however, to turn the argument around and begin by considering a consistent quantum theory, namely the string-like theory, and letting this theory define an ordering that makes it possible to define a consistent quantum membrane through unitary transformations.

Our starting point, therefore, will be to consider the $g=0$ theory where we simply have a string-like theory. Consider for simplicity the bosonic case. The supersymmetric case is completely analogous. Choose also, for definitness,

a closed membrane in the $\xi^{1}$-direction. The open case is treated in the same way. Solving the equations of motion, with periodic boundary conditions, one 
has quantum mechanically the string-like commutator for the left-moving modes

$$
\left[\alpha_{m}^{I}\left(\xi^{2}\right), \alpha_{n}^{J}\left(\xi^{2}\right)\right]=m \delta_{m,-n} \delta^{I J} \delta\left(\xi^{2}-\xi^{\prime 2}\right),
$$

and a corresponding one for the right-moving sector. The zeroth order groundstate of the membrane is given by the string-like groundstate $\alpha_{m}^{I}\left(\xi^{2}\right)\left|0, p_{0}\right\rangle_{0}=$ $p_{0}^{I} \delta_{m, 0}\left|0, p_{0}\right\rangle_{0}$ and $\tilde{\alpha}_{m}^{I}\left(\xi^{2}\right)\left|0, p_{0}\right\rangle_{0}=p_{0}^{I} \delta_{m, 0}\left|0, p_{0}\right\rangle_{0}$, for $p_{0}^{I}$ being a constant and $m \geq 0$. This implies that $\alpha_{m, n}^{I}\left|0, p_{0}\right\rangle_{0}=p_{0}^{I} \delta_{m, 0} \delta_{n, 0}\left|0, p_{0}\right\rangle_{0}$, for $m \geq 0$ and all $n$, etc., if we Fourier expand w.r.t. the $\xi^{2}$-coordinate. Next, let us construct a unitary operator, $U_{1}$, by

$$
U_{1} \equiv \exp \left(-i g:_{0} G_{1}:_{0}\right)
$$

Here $G_{1}$ is the generator constructed in section three, classically transforming away terms of order one of the membrane Hamiltonian, leaving the zeroth order string-like one. The notation :0 indicates a normal ordering w.r.t. the zeroth order groundstate i.e. the string-like one. We also order so that $q_{n}^{I}$ is on the left of $\alpha_{0, n}^{I}, n \neq 0$, and $q_{0}^{I}$ and $\alpha_{0,0}^{I}$ are Weyl ordered. In particular, $K$ defined in eq. (3.40), should be defined as an operator as

$$
K=\frac{1}{4}\left[k_{I} q_{0}^{I}\left(k_{I} \alpha_{0,0}^{I}\right)^{-1}+\left(k_{I} \alpha_{0,0}^{I}\right)^{-1} k_{I} q_{0}^{I}\right] .
$$

We now define a Hamiltonian

$$
H_{\text {membrane }}^{(1)} \equiv U_{1}:_{0} H_{\text {string }:_{0}} U_{1}^{\dagger} .
$$

In the classical limit, $H_{\text {membrane }}^{(1)}$ will become the membrane Hamiltonian in eq. (2.2) to order one in perturbation theory. Define the first order groundstate

$$
\left|0, p_{0}\right\rangle_{1}=U_{1}\left|0, p_{0}\right\rangle_{0}
$$

It satisfies $\alpha_{m, n}^{(1), I}\left|0, p_{0}\right\rangle_{1}=p_{0}^{I} \delta_{m, 0} \delta_{n, 0}\left|0, p_{0}\right\rangle_{1}$, for $m \geq 0$ and all $n$, where $\alpha_{m, n}^{(1), I} \equiv U_{1} \alpha_{m, n}^{I} U_{1}^{\dagger}$ 
Proceeding to second order, we take a unitary transformation

$$
U_{2} \equiv \exp \left(-i g^{2}:_{1} G_{2}:_{1}\right)
$$

where $:_{1}$ is normal ordering w.r.t. the first order groundstate. Define a Hamiltonian

$$
H_{\text {membrane }}^{(2)}=U_{2} U_{1}:_{0} H_{\text {string }}:_{0} U_{1}^{\dagger} U_{2}^{\dagger} .
$$

$H_{\text {membrane }}^{(2)}$ is classically equivalent to the membrane Hamiltonian to order two in perturbation theory. Using this procedure iteratively, one will arrive at a Hamiltonian that, in the classical limit, gives the membrane Hamiltonian to any order in perturbation theory. This construction of the membrane Hamiltonian gives a specific ordering of the operators, that from a membrane point of view is non-trivial. In Appendix B a toy model with similar properties is treated. The treatment gives the explicit construction up to second order.

The groundstate that one gets to order $N$ is

$$
\left|0, p_{0}\right\rangle_{N}=U_{N} \ldots U_{1}\left|0, p_{0}\right\rangle_{0}
$$

which satisfies that

$$
\alpha_{m, n}^{(N), I}\left|0, p_{0}\right\rangle_{N}=p_{0}^{I} \delta_{m, 0} \delta_{n, 0}\left|0, p_{0}\right\rangle_{N}
$$

for $m \geq 0$ and all $n$, where

$$
\alpha_{m, n}^{(N), I}=U_{N} \ldots U_{1} \alpha_{m, n}^{I} U_{N}^{\dagger} \ldots U_{1}^{\dagger}
$$

Note that $:_{0} H_{\text {string }}:_{0}\left|0, p_{0}\right\rangle_{N}$, is, in general, infinite for $N \geq 1$. This implies that the perturbation in $g$ is non-perturbative from a string point of view. Note also that $K$ is a well-defined operator in eq. (5.8) for $p_{0}^{I} \neq 0$. Eigenstates to the Hamiltonian may also be constructed in the same fashion. Let

$$
|\phi\rangle_{0}=\hat{\phi}\left|0, p_{0}\right\rangle_{0}
$$


be any string-like eigenstate. Then, obviously,

$$
|\phi\rangle_{N}=U_{N} \ldots U_{1} \hat{\phi}\left|0, p_{0}\right\rangle_{0}
$$

is an eigenstate to the Hamiltonian to order $N$ in perturbation theory. $|\phi\rangle_{0}$ consists of all states given by applying the creation operators $\alpha_{-m, n}^{I}, \tilde{\alpha}_{-m, n}^{I}$ and $\exp \left(i \sum_{n} \tilde{k}_{-n, I} q_{n}^{I}\right)$, for $m>0$, all $n$ and constant $\tilde{k}_{n, I}$, to the groundstate such that eq. (3.20) is satisfied.

Our theory is obviously invariant under the $(D-3)$-dimensional Lorentz group. But, in addition, one has $(D-1)$-dimensional Lorentz symmetry provided $D-1=26$ or $D-1=10$ i.e. $D=27$ and $D=11$, respectively. This follows trivially from the fact that the unperturbed string-like theory has this unbroken symmetry for the critical dimensions and the unitary equivalence of stretched membranes to string-like configurations at any order in perturbation theory.

This does not, however, prove the existence of a consistent quantum membrane theory with 27- or 11-dimensional Lorentz invariance. The commutators that need to be checked for the closure of full Lorentz algebra are those which involve at least one of $M^{D-2, I}, M^{D-2,+}$ and $M^{D-2,-}, I=1, \ldots 24$ or $I=1, \ldots 8$. These generators are, for the bosonic case and at the classical level, of the form

$$
\begin{aligned}
M^{D-2, I} & =\frac{1}{\sqrt{g}}\left[\int d^{2} \xi P^{I} \xi^{2}+g \int d^{2} \xi X^{I} B\right] \\
M^{D-2,+} & =\frac{1}{\sqrt{g}}\left[2 \pi^{2}+g \xi^{0} \int d^{2} \xi B\right] \\
M^{D-2,-} & =\frac{1}{\sqrt{g}}\left[-\int d^{2} \xi \mathcal{H} \xi^{2}+g \int d^{2} \xi X_{0}^{-} B\right],
\end{aligned}
$$

where $H=\int d^{2} \xi \mathcal{H}, P_{0}^{U}=\int d^{2} \xi \mathcal{P}^{U}$ etc. $X^{-}$is solved from the constraints and contains terms of zeroth order as well as first order terms in $g$. If one computes the commutators, at the quantum level and up to order $\mathcal{O}\left(g^{-1 / 2}\right)$, it is straightforward to see that they are anomaly free. To next order, terms 
of order $g^{0}$, the calculation is complicated since different orders will be mixed. Furthermore, the unitary transformations will not simplify the calculations. The quantum consistency of the full Lorentz algebra is, therefore, still an open and non-trivial question.

Let us now discuss the mass spectrum that is implied by our construction. The unitary transformations, that transform the membrane states into string-like states, ensure that, from a $(D-1)$-dimensional point of view, the mass spectrum will be exactly the same as for string theory. There will, however, be an infinite degeneracy corresponding to the $\xi^{2}$-dependence. From a $D$-dimensional point of view the mass spectrum is slightly different. The mass is determined from the operator

$$
\begin{aligned}
m^{2} & =-P_{0}^{U} P_{U, 0}+a \\
& =-2 P_{0}^{+} P_{0}^{-}-P_{0}^{I} P_{0, I}-P_{0}^{D-2} P_{0}^{D-2}+a \\
& =2 H-P_{0}^{I} P_{0, I}-P_{0}^{D-2} P_{0}^{D-2}+a,
\end{aligned}
$$

where $a$ is the conventional constant introduced because of ordering ambiguities. For the bosonic membrane we have $a=-4$ and $a=-2$ for the closed and open cases, respectively, which follows from a generalization of the usual string argument 22]. For the space-time supersymmetric membrane one has $a=0$ for both cases. From eqs. (3.8) and (4.8) we have $P_{0}^{D-2}=-\sqrt{g} \int d^{2} \xi B$ and $P_{0}^{D-2}=-\sqrt{g} \int d^{2} \xi \tilde{B}$ for the bosonic and supermembrane, respectively.

For $g=0$ we have that the mass-shell condition w.r.t. the $(D-1)$-dimensional Poincaré group and the $D$-dimensional one are equivalent. We find

$$
\left(m^{(0)}\right)^{2} \equiv 2 H_{0}-P_{0}^{I} P_{0, I}+a=m^{2}+\mathcal{O}(g)
$$

and a discrete mass-spectrum. If we now apply unitary transformations to order 
$N$ we will get

$$
\left(m^{(N)}\right)^{2} \equiv 2 H_{N}-P_{0}^{I} P_{0, I}+a=m^{2}+P_{0}^{(N), D-2} P_{0}^{(N), D-2}+\mathcal{O}\left(g^{N+1}\right),
$$

so that

$$
(m)^{2}=\left(m^{(N)}\right)^{2}-P_{0}^{(N), D-2} P_{0}^{(N), D-2}+\mathcal{O}\left(g^{N+1}\right) .
$$

The mass spectrum is, therefore, given by string spectrum corrected by the $(D-$ 2)-component of the momentum. Concentrating on the bosonic membrane, we have $B_{0}^{(N)}=-\frac{1}{\sqrt{g}} P_{0}^{(N), D-2}$ and we find, by eq. (3.19),

$$
\begin{aligned}
B_{0}^{(N)} & \equiv \int d^{2} \xi B^{(N)} \\
& =-\sum_{(a)}\left[i n q_{(-a)}^{(N)} \alpha_{0,(a)}^{(N)}\right. \\
& \left.+\sum_{m>0,(a)} \frac{n}{m}\left(\alpha_{-m,(-a)}^{(N)} \alpha_{m,(a)}^{(N)}+\tilde{\alpha}_{-m,(-a)}^{(N)} \tilde{\alpha}_{m,(a)}^{(N)}\right)\right] .
\end{aligned}
$$

Note that one could, in principle, add a normal ordering constant to $B_{0}^{(N)}$. Evaluating it by a zeta-function regularization yields the value zero. Evaluating the commutator of this term with $q_{n}^{(N), I}, \alpha_{m, n}^{(N), I}$ and $\tilde{\alpha}_{m, n}^{(N), I}$ gives that this operator counts the mode number w.r.t. the $\xi^{2}$-direction,

$$
\begin{aligned}
{\left[B_{0}^{(N)}, q_{n}^{(N)}\right] } & =n q_{n}^{(N)} \\
{\left[B_{0}^{(N)}, \alpha_{m, n}^{(N), I}\right] } & =n \alpha_{m, n}^{(N), I} \\
{\left[B_{0}^{(N)}, \tilde{\alpha}_{m, n}^{(N), I}\right] } & =n \tilde{\alpha}_{m, n}^{(N), I} .
\end{aligned}
$$

Furthermore, $B_{0}^{(N)}\left|0, p_{0}\right\rangle_{N}=0$. The operators $q_{n}$ need to be applied in the form $\exp \left(i \sum_{n} \tilde{k}_{-n, I} q_{n}^{I}\right)$ to get an eigenstate of the Hamiltonian. The resulting state is, however, not an eigenstate of $B_{0}^{(N)}$ unless $\tilde{k}_{n}=0$, for $n \neq 0$. We restrict 
ourselves, therefore, to $\tilde{k}_{n}=0$ for $n \neq 0$. Using the result in eq. (5.17) one will find

$$
(m)^{2}=\left(m^{(N)}\right)^{2}-g n^{2}+\mathcal{O}\left(g^{N+1}\right)
$$

where $n$ is the mode number of the eigenstate in the $\xi^{2}$-direction. Thus we see that the degeneracy of the excited states will be lifted and the mass spectrum will be split due to the mode number in the $\xi^{2}$-direction. The splitting is exact to all orders and independent of the order of the perturbation. The groundstate mass is not corrected. As can be seen from eq. (5.20), we still have massless string-like states at the first excited level with $n=0$. One would expect that when $g n^{2} \sim 1$ the perturbation theory is not valid. This implies that our perturbation theory would be valid for $|n|<\frac{1}{\sqrt{g}}$ that is, for fixed $g$, the magnitude of the mode number in the $\xi^{2}$-direction cannot take arbitrarily large values.

For the supermembrane, $B_{0}^{(N)}$ is replaced by $\tilde{B}_{0}^{(N)}$, which again counts the modes in the $\xi^{2}$-direction. Therefore, we get exactly the same result for the mass spectrum, i.e. the splitting is of the same form as above, where $n$ now also includes fermionic excitations. In particular, the mass of the groundstate will not get any corrections and we will have massless states.

Note that for a closed membrane in the $\xi^{2}$-direction, we will get consistency conditions on the eigenvalues of $P_{0}^{D-2}$ due to the gauge choice, eq. (3.6). This choice is consistent for closed membranes in the $\xi^{2}$-direction only if $X_{0}^{D-2}$ is periodic. Since $\xi^{2}$ is periodic with period $2 \pi$ we have $X_{0}^{D-2}$ is periodic with period $2 \pi / \sqrt{g}$. Consequently, $P_{0}^{D-2}$ is quantized and take values $n \sqrt{g}, n \in \mathcal{Z}$. Thus, from eq. (5.14) one will get

$$
m^{2}=2 H-P_{0}^{I} P_{0}^{I}-g n^{2}+a .
$$

This is exactly the same spectrum as above. It is a non-trivial check of consistency 
that these two independent derivations are in agreement with each other. It also shows that the magnitude of the splitting is non-perturbative.

One may repeat the analysis for an open membrane or semi-open membrane. This is straightforward and the spectrum will be of the same principal form. In particular, it will contain massless states.

As mentioned in section three, we have set $f\left(\xi^{2}\right)=0$, which by consistency, restricts the choices of boundary conditions. Let us briefly consider the case when $f$ is non-zero. Upon quantization, the function will become an operator $\hat{f}$ depending, in general, on the basic operators $\alpha_{n, m}^{I}$ and $q_{n}^{I}$. In order to determine the spectrum one needs to diagonalize $B_{0}$. The resulting eigenvalues will, therefore, depend on $\int d^{2} \xi \hat{f}$ and may result in a discrete or continous spectrum. If we, for simplicity, assume that $\int d^{2} \xi \hat{f}$ is diagonal for the same set of states as with $\hat{f}=0$, then the eigenvalues, $f_{0}$, of $\int d^{2} \xi \hat{f}$ will have the effect that $B_{0}$ is changed into $B_{0}+f_{0}$. Therefore, the spectrum in eq. (5.20) is shifted to

$$
m^{2}=\left(m^{(N)}\right)^{2}-g\left(n+2 \pi f_{0}^{(M)}\right)^{2},
$$

where $(M)$ is a collection of quantum numbers labeling the states.

For closed membranes in the $\xi^{2}$-direction we still have to fulfill the quantization condition of the momentum, discussed above. In this case, the resulting spectrum is simply shifted. For the supermembrane, this will imply that there are no massless states, which in turn implies that supersymmetry is spontaneously broken. For the bosonic case, one cannot rule this out. The difference here is that massless states for the unperturbed theory are excited states. Then it may be possible to have $n+2 \pi f_{0}^{(M)}=0$.

For the open case, the situation may be very different. First of all, the spectrum may either be continous or discrete, depending on $\hat{f}$. Secondly, even for a discrete spectrum, $2 \pi f_{0}^{(M)}$ may not be an integer. The simplest case is when 
$\hat{f}=C$ is a constant. The groundstate will have $m^{2}$ that is shifted by a constant value, $-g(2 \pi)^{4} C^{2}$. If this value is an integer then the spectrum of the excited states will remain the same. The energies of the eigenstates will be permuted within a multiplet. Massless states are only possible for integer eigenvalues of $2 \pi f_{0}^{(M)}$, and only for the bosonic case, as discussed above.

\section{Conclusions}

We have in this paper shown that stretched membrane configurations are essentially described by string-like excitations. We have suggested that these stringlike modes could play the role of elementary membrane states. There are many interesting and important questions that need to be answered. First of all the question of consistency of the perturbation theory, e.g. the convergence, as well as the full 27- or 11-dimensional Lorentz symmetry.

One would also like to probe the non-perturbative properties. If the conjecture that the original membrane action describes a second quantized theory, then it is natural to expect that non-perturbative effects will include interaction among the string-like states. We hope to be able to come back to this important question in the future.

Finally, it would be interesting and important to further study the matrix model calculations in the light of our presented results.

Acknowledgement. We are indebted to Anders Westerberg for helpful discussions and comments on the manuscript. 


\section{A Lightcone coordinate conventions}

$$
\begin{aligned}
A^{+} & =\frac{1}{\sqrt{2}}\left(A^{0}+A^{D-1}\right) \\
A^{-} & =\frac{1}{\sqrt{2}}\left(-A^{0}+A^{D-1}\right) \\
A_{U} B^{U} & =A^{+} B^{-}+A^{-} B^{+}+A_{I} B_{I}+A_{D-2} B_{D-2},
\end{aligned}
$$

where $U=1, \ldots, D-1$ and $I=1, \ldots, D-3$. We use this explicit basis of the gamma matrices

$$
\begin{aligned}
& \Gamma^{+}=\mathbb{1}_{16} \otimes\left(\begin{array}{cc}
0 & 0 \\
\sqrt{2} i & 0
\end{array}\right) \\
& \Gamma^{-}=\mathbb{1}_{16} \otimes\left(\begin{array}{cc}
0 & \sqrt{2} i \\
0 & 0
\end{array}\right) \\
& \Gamma_{A}=\gamma_{A} \otimes\left(\begin{array}{cc}
1 & 0 \\
0 & -1
\end{array}\right) .
\end{aligned}
$$

where $A=1, \ldots, D-2$ and $\gamma_{9}$ can be taken to be

$$
\gamma_{9}=\left(\begin{array}{cc}
\mathbb{1}_{8} & 0 \\
0 & -\mathbb{1}_{8}
\end{array}\right) .
$$

The $\bar{\theta}$ is defined by

$$
\bar{\theta}=\theta^{t} \Gamma^{0}
$$

which in our basis is

$$
\begin{aligned}
& \bar{\theta}^{2 \beta-1}=i \theta^{2 \beta} \\
& \bar{\theta}^{2 \beta}=-i \theta^{2 \beta-1} \quad \beta=1, \ldots, 16
\end{aligned}
$$




\section{B A toy model}

Let us show how our construction works for a toy model that captures many of the features of the membrane. In particular, it will expose the non-trivial ordering one gets by our construction. The theory is defined by

$$
\begin{aligned}
H & =H_{0}+g H_{1} \\
H_{0} & =\frac{1}{2} p^{2}+a^{\dagger} a \\
H_{1} & =\left(a^{\dagger}\right)^{3} a+a^{\dagger}(a)^{3},
\end{aligned}
$$

with the following non-zero Poisson brackets

$$
\begin{aligned}
\{q, p\} & =1 \\
\left\{a, a^{\dagger}\right\} & =-i .
\end{aligned}
$$

Let us construct, order by order, the canonical transformation which maps $H_{0}$ to $H_{1}$. To first order we need to solve

$$
\begin{aligned}
H_{(1)} & \equiv \exp \left(-g \operatorname{ad}_{G_{1}}\right) H_{0} \\
& =H_{0}+g\left\{H_{0}, G_{1}\right\}+\frac{g^{2}}{2}\left\{\left\{H_{0}, G_{1}\right\}, G_{1}\right\} \\
& =H+\mathcal{O}\left(g^{2}\right) .
\end{aligned}
$$

One easily finds the following solution

$$
G_{1}=\frac{i}{2}\left[\left(a^{\dagger}\right)^{3} a-a^{\dagger}(a)^{3}\right] .
$$


Defining new coordinates by the canonical transformation, one will get

$$
\begin{aligned}
q_{(1)} & =\exp \left(-g \operatorname{ad}_{G_{1}}\right) q=q \\
p_{(1)} & =\exp \left(-g \operatorname{ad}_{G_{1}}\right) p=p \\
a_{(1)} & =\exp \left(-g \operatorname{ad}_{G_{1}}\right) a=a+\frac{1}{2} g\left(3\left(a^{\dagger}\right)^{2} a-a^{3}\right)+\frac{1}{8} g^{2}\left(3\left(a^{\dagger}\right)^{4} a+6\left(a^{\dagger}\right)^{2} a^{3}\right. \\
& \left.+3 a^{5}\right) \\
a_{(1)}^{\dagger} & =\exp \left(-g \operatorname{ad}_{G_{1}}\right) a^{\dagger}=a^{\dagger}+\frac{1}{2} g\left(3\left(a^{\dagger}\right) a^{2}-\left(a^{\dagger}\right)^{3}\right)+\frac{1}{8} g^{2}\left(3 a^{\dagger} a^{4}+6\left(a^{\dagger}\right)^{3} a^{2}\right. \\
& \left.+3\left(a^{\dagger}\right)^{5}\right) .
\end{aligned}
$$

In terms of the new coordinates, the Hamiltonian $H_{(1)}$ has the same form as $H_{0}$. Expanding in the old coordinates yields

$$
\begin{aligned}
H_{(1)} & =\frac{1}{2} p_{(1)}^{2}+a_{(1)}^{\dagger} a_{(1)} \\
& =H\left(p, a, a^{\dagger}\right)+4 g^{2}\left(a^{\dagger}\right)^{3} a^{3}+\mathcal{O}\left(g^{3}\right) \\
& =H\left(p, a, a^{\dagger}\right)+4 g^{2}\left(a_{(1)}^{\dagger}\right)^{3} a_{(1)}^{3}+\mathcal{O}\left(g^{3}\right),
\end{aligned}
$$

where we in the last line have used eq. (B.14). The $g^{2}$-term can be transformed away with an additional canonical transformation

$$
\exp \left(-g^{2} \operatorname{ad}_{G_{2}}\right) H_{(1)}=H\left(p, a, a^{\dagger}\right)+\mathcal{O}\left(g^{3}\right)
$$

The result one finds is

$$
G_{2}=4 K_{(1)}\left(a_{(1)}^{\dagger}\right)^{3} a_{(1)}^{3}
$$

where

$$
K_{(1)}=\frac{1}{2}\left(q_{(1)} p_{(1)}^{-1}+p_{(1)}^{-1} q_{(1)}\right)
$$

and satisfies

$$
\left\{H_{(1)}, K_{(1)}\right\}=-1 \text {. }
$$


We have now constructed the infinitesimal canonical generators that mapsthe unperturbed Hamiltonian to the perturbed one to order two in perturbation theory. This we have done classically. Let us now follow the procedure outlined in section five to construct a well-defined quantum Hamiltonian by applying infinitesimal unitary transformations to the unperturbed quantum version of $H_{0}$. The Poisson brackets are exchanged with the commutators

$$
\begin{aligned}
{[q, p] } & =i \\
{\left[a, a^{\dagger}\right] } & =1 .
\end{aligned}
$$

To zeroth order in perturbation theory one can define a groundstate by

$$
\begin{aligned}
a\left|0, p_{0}\right\rangle_{0} & =0 \\
p\left|0, p_{0}\right\rangle_{0} & =p_{0}\left|0, p_{0}\right\rangle_{0} .
\end{aligned}
$$

From this groundstate one can now construct the states of this theory

$$
\begin{aligned}
\left|N, p_{0}\right\rangle & =\frac{1}{\sqrt{N !}}\left(a^{\dagger}\right)^{N}\left|0, p_{0}\right\rangle \\
\left|0, p_{0}+k_{0}\right\rangle & =\exp \left(i k_{0} q\right)\left|0, p_{0}\right\rangle,
\end{aligned}
$$

where $k_{0}$ is a constant. Let us make a unitary transformation, up to order two in $g$, constructed from $G_{1}$ in eq. (B.13),

$$
U_{1} \equiv \exp \left(i g:_{0} G_{1}:_{0}\right)=1+i g:_{0} G_{1}:_{0}-\frac{1}{2} g^{2}\left(:_{0} G_{1}:_{0}\right)^{2}+\mathcal{O}\left(g^{3}\right)
$$

We use this transformation to define a new groundstate as

$$
\begin{aligned}
\left|0, p_{0}\right\rangle_{1} & =\exp \left(i g:_{0} G_{1}:_{0}\right)\left|0, p_{0}\right\rangle_{0} \\
& =\left(1-\frac{g}{2}\left[\left(a^{\dagger}\right)^{3} a-a^{\dagger}(a)^{3}\right]+\frac{g^{2}}{8}\left[\left(a^{\dagger}\right)^{3} a-a^{\dagger}(a)^{3}\right]\right. \\
& \left.\times\left[\left(a^{\dagger}\right)^{3} a-a^{\dagger}(a)^{3}\right]\right)\left|0, p_{0}\right\rangle_{0}+\mathcal{O}\left(g^{3}\right)=\left|0, p_{0}\right\rangle_{0} .
\end{aligned}
$$


Therefore, the first order groundstate is the same as the zeroth order one ${ }^{5}$. Let us also transform the operators

$$
\begin{aligned}
q_{(1)} & \equiv U_{1} q U_{1}^{\dagger}=q \\
p_{(1)} & \equiv U_{1} p U_{1}^{\dagger}=p \\
a_{(1)} & \equiv U_{1} a U_{1}^{\dagger}=a+\frac{1}{2} g\left(3\left(a^{\dagger}\right)^{2} a-a^{3}\right)+\frac{1}{8} g^{2}\left(3\left(a^{\dagger}\right)^{4} a+3\left(a^{\dagger}\right)^{2} a^{3}\right. \\
& \left.+6 a^{\dagger} a^{2} a^{\dagger} a-3 a^{2}\left(a^{\dagger}\right)^{2} a+3 a^{5}\right) \\
a_{(1)}^{\dagger} & \equiv U_{1} a^{\dagger} U_{1}^{\dagger}=a^{\dagger}+\frac{1}{2} g\left(3 a^{\dagger} a^{2}-\left(a^{\dagger}\right)^{3}\right)+\frac{1}{8} g^{2}\left(3 a^{\dagger} a^{4}+3\left(a^{\dagger}\right)^{3} a^{2}\right. \\
& \left.+6 a^{\dagger} a\left(a^{\dagger}\right)^{2} a-3 a^{\dagger} a^{2}\left(a^{\dagger}\right)^{2}+3\left(a^{\dagger}\right)^{5}\right) .
\end{aligned}
$$

Inserting this, one gets

$$
\begin{aligned}
H_{(1)} & =\frac{1}{2} p_{(1)}^{2}+a_{(1)}^{\dagger} a_{(1)} \\
& =\frac{1}{2} p^{2}+a^{\dagger} a+g\left(\left(a^{\dagger}\right)^{3} a+a^{\dagger} a^{3}\right)+g^{2}\left(\left(a^{\dagger}\right)^{3} a^{3}+\frac{3}{2} a^{\dagger} a^{2}\left(a^{\dagger}\right)^{2} a\right. \\
& \left.+\frac{3}{4}\left(a^{\dagger}\right)^{2} a^{2} a^{\dagger} a+\frac{3}{4} a^{\dagger} a\left(a^{\dagger}\right)^{2} a^{2}\right)+\mathcal{O}\left(g^{3}\right) \\
& =H+g^{2}\left(\left(a_{(1)}^{\dagger}\right)^{3} a_{(1)}^{3}+\frac{3}{2} a_{(1)}^{\dagger} a_{(1)}^{2}\left(a_{(1)}^{\dagger}\right)^{2} a_{(1)}+\frac{3}{4}\left(a_{(1)}^{\dagger}\right)^{2} a_{(1)}^{2} a_{(1)}^{\dagger} a_{(1)}\right. \\
& \left.+\frac{3}{4} a_{(1)}^{\dagger} a_{(1)}\left(a_{(1)}^{\dagger}\right)^{2} a_{(1)}^{2}\right)+\mathcal{O}\left(g^{3}\right) .
\end{aligned}
$$

Thus, to first order, the quantum Hamiltonians $H$ and $H_{(1)}$ coincide.

Let us now use $G_{2}$, given by eq. (B.17), to construct the next unitary transformation,

$$
U_{2}=\exp \left(i g^{2}:_{1} G_{2}:_{1}\right)
$$

\footnotetext{
${ }^{5}$ This is not true for more general models, for example, where $H_{1}$ contains terms of the form $\left(a^{\dagger}\right)^{4}+a^{4}$.
} 
We then define the Hamiltonian

$$
\begin{aligned}
H_{(2)} & \equiv \frac{1}{2} p_{(2)}^{2}+a_{(2)}^{\dagger} a_{(2)}=U_{2} H_{(1)} U_{2}^{\dagger} \\
& =H+g^{2}\left(\left(a_{(1)}^{\dagger}\right)^{3} a_{(1)}^{3}+\frac{3}{2} a_{(1)}^{\dagger} a_{(1)}^{2}\left(a_{(1)}^{\dagger}\right)^{2} a_{(1)}+\frac{3}{4}\left(a_{(1)}^{\dagger}\right)^{2} a_{(1)}^{2} a_{(1)}^{\dagger} a_{(1)}\right. \\
& \left.+\frac{3}{4} a_{(1)}^{\dagger} a_{(1)}\left(a_{(1)}^{\dagger}\right)^{2} a_{(1)}^{2}-4\left(a_{(1)}^{\dagger}\right)^{3} a_{(1)}^{3}\right)+\mathcal{O}\left(g^{3}\right)
\end{aligned}
$$

As can be seen from this equation, the Hamiltonian $H^{(2)}$ differs from $H$, up to order two, only by ordering terms. The groundstate to this order, $\left|0, p_{0}\right\rangle_{2}=$ $U_{2}\left|0, p_{0}\right\rangle_{1}$, is the same as the zeroth order vacuum. The operators are transformed to

$$
\begin{aligned}
& q_{(2)}=U_{2} q_{(1)} U_{2}^{\dagger}=q_{(1)}+2 g^{2}\left(q_{(1)} p_{(1)}^{-2}+p_{(1)}^{-2} q_{(1)}\right)\left(a_{(1)}^{\dagger}\right)^{3}\left(a_{(1)}\right)^{3} \\
& p_{(2)}=U_{2} p_{(1)} U_{2}^{\dagger}=p_{(1)}+4 g^{2} p_{(1)}^{-1}\left(a_{(1)}^{\dagger}\right)^{3}\left(a_{(1)}\right)^{3} \\
& a_{(2)}=U_{2} a_{(1)} U_{2}^{\dagger}=a_{(1)}-12 g^{2} i K_{(1)}\left(a_{(1)}^{\dagger}\right)^{3}\left(a_{(1)}\right)^{2} \\
& a_{(2)}^{\dagger}=U_{2} a_{(1)}^{\dagger} U_{2}^{\dagger}=a_{(1)}+12 g^{2} i K_{(1)}\left(a_{(1)}^{\dagger}\right)^{2}\left(a_{(1)}\right)^{3},
\end{aligned}
$$

where $q_{(1)}, p_{(1)}, a_{(1)}$ and $a_{(1)}^{\dagger}$ are given by eq. (B.25). As can be seen from the equations, even if the theory is simple and we only consider the transformations up to order two, the resulting expressions are relatively complicated.

\section{References}

[1] P. K. Townsend, Phys. Lett. B 350 (1995) 184 arXiv:hep-th/9501068.

[2] E. Witten, Nucl. Phys. B 443 (1995) 85 arXiv:hep-th/9503124.

[3] J. Goldstone, unpublished

[4] J. Hoppe, Quantum theory of a massless Relativistic Surface and a twodimensional bound state problem, $\mathrm{PhD}$ thesis MIT 1982 
[5] B. de Wit, J. Hoppe and H. Nicolai, Nucl. Phys. B 305, [FS23] (1988) 545.

[6] T. Banks, W. Fischler, S. H. Shenker and L. Susskind, Phys. Rev. D 55 (1997) 5112 arXiv:hep-th/9610043.

[7] N. Seiberg, Phys. Rev. Lett. 79 (1997) 3577

[8] A. Sen, Adv. Theor. Math. Phys.2 (1998) 51

[9] J. Björnsson and S. Hwang, Nucl. Phys. B 689 (2004) 37 arXiv:hep-th/0403092.

[10] U. Lindström and R. von Unge, Phys. Lett. B 403, 233 (1997) arXiv:hep-th/9704051.

[11] M. J. Duff, P. S. Howe, T. Inami and K. S. Stelle, Phys. Lett. B 191 (1987) 70.

[12] I. Bars, C. N. Pope and E. Sezgin, Phys. Lett. B 198 (1987) 455.

[13] I. Bars, Nucl. Phys. B 308, 462 (1988).

[14] K. Fujikawa and J. Kubo, Phys. Lett. B 199, 75 (1987).

[15] U. Marquard and M. Scholl, Phys. Lett. B 227 (1989) 227.

[16] U. Marquard, R. Kaiser and M. Scholl, Phys. Lett. B 227 (1989) 234.

[17] B. Simon, Annals Phys. 146 (1983) 209.

[18] B. de Wit, M. Luscher and H. Nicolai, Nucl. Phys. B 320 (1989) 135.

[19] I. Y. Aref'eva, A. S. Koshelev and P. B. Medvedev, Nucl. Phys. B 579 (2000) 411 arXiv:hep-th/9911149.

[20] P. A. M. Dirac, Proc. Roy. Soc. A268 57 (1962)

[21] E. Bergshoeff, E. Sezgin and P. K. Townsend, Phys. Lett. B 189 (1987) 75.

[22] L. Brink and H. B. Nielsen, Phys. Lett. B 45, 332 (1973). 